\title{
Application of optimization methods for improved electrical metrology
}

\author{
Marija Cundeva-Blajer \\ Ss. Cyril and Methodius University, Faculty of Electrical Engineering and Information Technologies, ul. Ruger Boskovic br. 18, POBox 574, \\ 1000 Skopje, R. Macedonia
}

ABSTRACT

In electrical quantities metrology numerous examples of stochastic processes exist and a need for optimal solutions is posed. Here stochastic genetic algorithm optimization is used for solving two typical metrological problems: 1) minimization of the metrological parameters (final accuracy) in the design process of instruments and 2) predicting metrological reference standard's time-drift, i.e. recalibration interval. The first case is the optimal metrological design of a combined instrument transformer and the second case is the analysis of resistance standard time-drift.

Section: RESEARCH PAPER

Keywords: optimization; genetic algorithm; electrical measurements; instrument transformer; resistance standard

Citation: Marija Cundeva-Blajer, Application of optimization methods for improved electrical metrology, Acta IMEKO, vol. 5, no. 3, article 3, November 2016, identifier: IMEKO-ACTA-05 (2016)-03-03

Section Editor: Franco Pavese, Italy

Received April 18, 2016; In final form April18, 2016; Published November 2016

Copyright: (C) 2016 IMEKO. This is an open-access article distributed under the terms of the Creative Commons Attribution 3.0 License, which permits unrestricted use, distribution, and reproduction in any medium, provided the original author and source are credited

Corresponding author: Marija Cundeva-Blajer, e-mail: mcundeva@feit.ukim.edu.mk

\section{INTRODUCTION}

In metrology among other problems two of them represent general challenges [1]:

1. Reduction of errors and uncertainties during the design process of measurement instrumentation and devices;

2. Prediction of the time-behaviour of measurement instrumentation or standards, i.e. estimation of the optimal recalibration interval.

Construction of functions based on experimental data is a widespread and important procedure in measurement problems (e.g. fitting of calibration curves for measuring instruments, or deduction of equations for indirect measurement methods) [2]. In these cases the quality of the empirical function is essential for ensuring the integral quality of measurement. The empirical function must be sufficiently precise for practical purposes and its estimates of the accuracy characteristics must be reliable. The traditional method for fitting of functions is the classical least squares (LS) method. The LS estimates are optimal only under strict conditions upon errors of data, that is, within a classic regression model. So, when it does not hold in practice, some other methods are required, so-called confluent methods. However, a general confluent model is not appropriate for practice either, since neither LS nor other data processing methods can provide statistically consistent estimates for functions. Therefore, the key problem is to construct proper extensions of regression models, which allow obtaining consistent estimates for functions [2]. The proper model should include additional information on data or data errors, which makes it possible to construct consistent estimates. Confluent estimates should be constructed in accordance with the type of the extended model. However, the most important problem for measurements is to study the accuracy characteristics of these estimates, taking into account both random and systematic components of data errors. In [2], several ways for expansion of regression models are outlined, and associated groups of estimates are studied. The accuracy properties of estimates are considered, which may be compared with the corresponding characteristics of classical LS estimates. This is a heuristic approach, but the optimisation does not work in this case. The main criterion for deriving confluent methods is the consistency as an asymptotic property of estimates. However, confluent estimates are used in the cases of small or modest volumes of data. Therefore, the accuracy characteristics of various confluent estimates should be also compared with the classical LS fitting under practical conditions [2]. 
In electrical measurements numerous examples of stochastic processes exist, like the electrical quantities standards timebehaviour, [1], [3], [4], [5], and [6].

In recent years the application of stochastic optimization tools for the design of electrical devices (including electrical instrumentation) is evident [10]-[13].

In electrical power distribution grids, state estimation is the main part of the supervisory control and data acquisition (SCADA) system. The aim is to determine an estimate of the system state based on a network model and the measurements available. The network state consists of voltage bus magnitudes and angles, line flows, loads, transformer taps, active and reactive power, power injections and generator outputs. In a typical state estimation scenario, the measurement data includes active and reactive power flows, power injections and voltage magnitudes at the bus. In [3] a quasi-dynamic state estimation method, based on a disturbance observer is proposed. Its aim is to reconstruct electrical power (active and reactive) from available measurements. On the distribution level, where a high number of nodes is accompanied by a small number of measurements, the typical approach is to replace missing data with so-called pseudo-measurements. However, in this way the state estimation quality depends on the reliability of the pseudomeasurements. That means that in order to improve the accuracy and reliability of the state estimation result, improved pseudo-measurement models are needed. In [3] stochastic models to obtain reliable pseudo-measurements and to improve state estimation results are investigated. The autoregressive moving average models (ARMA) to model the error of pseudomeasurements are used. The employed disturbance observer state estimation method is then able to improve the quality of the pseudo-measurements based on assumed ARMA process coefficients.

The optimal measurement theory arose as a branch of the inverse problems theory [4]. The mathematical justification of the algorithm for numerical restoration of the signal distorted by inertia and resonances in measuring transducers is presented in [4]. One of the directions for the development of this theory is mathematical justification of restoration of stochastic signals, in particular"white noise".

Design applications generally aim to minimise manufacturing or material costs subject to performance criteria, which is an inverse problem requiring optimisation techniques [7]. What mathematical physics denotes as inverse problems is the class of problems which are fundamental in measurement theory and practice [8]. The main objective of such problems is to develop procedures for acquiring information on object and phenomena, accompanied by decreasing the distortion caused by measuring instruments [8]. In [7] the implementation of Levenburg-Marquardt, sequential quadratic programming, Nelder-Mead, simulated annealing and practical swarm optimisation for metrology and design with continuous models is reported. In [10]-[13] the genetic algorithm (GA) as stochastic optimization method is applied for optimal design.

Calibration consists of two stages [15]. In stage 1 a relation is established between values provided by measurement standards and corresponding instrument response values. In stage 2 this relation is used to obtain measurement results from further response values. Polynomials of various degrees, determined by least squares, are extensively used as empirical calibration functions in metrology [9]. In this contribution an original computer program with embedded genetic algorithm developed at the Ss. Cyril and Methodius University in Skopje [6] is used for solving two metrological problems:

a) Metrological optimal design of a combined instrument transformer;

b) Predictions of resistance standards time drift for the determination of the optimal recalibration period, [16].

\section{DESCRIPTION OF GENETIC ALGORITHM PROGRAM FOR METROLOGICAL OPTIMIZATION}

On one side the concept of a genetic algorithm is very simple as displayed in Figure 1, but on the other side it is a very powerful and robust computational tool.

The GA implemented in the original computer program developed at the Ss. Cyril and Methodius University in Skopje

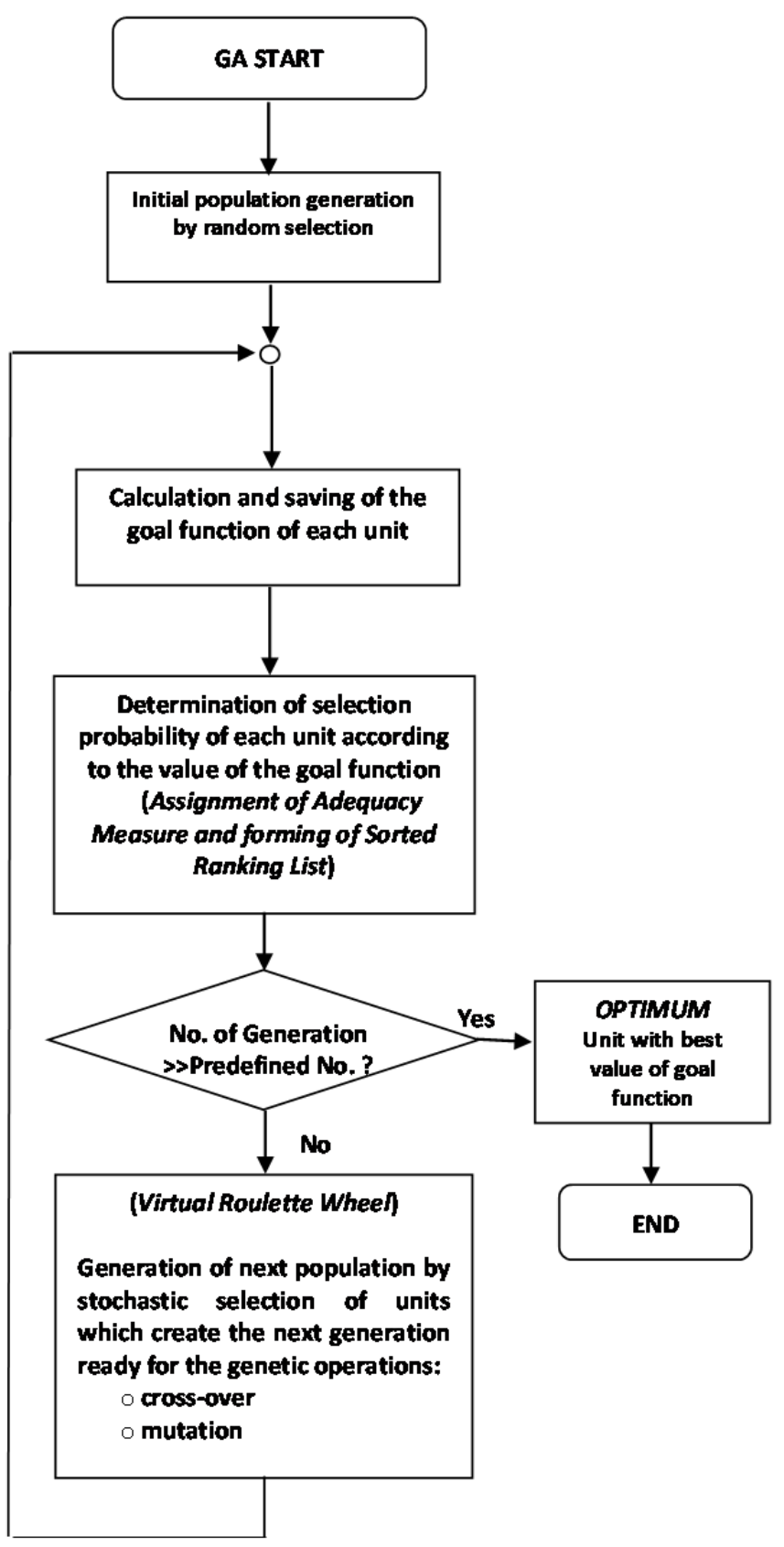

Figure 1. Genetic algorithm for solving problems of stochastic nature in metrology of electrical quantities. 
[10] starts with the initial units (chromosomes) stochastic generation. Each chromosome is composed of the same number of genes. It is the initial population (generation).

The size of the population is between 20 and 100. Iteration in the GA is the cycle of estimation, selection and reproduction of the population. Each unit represents the possible optimal solution. A possible solution for each unit is estimated according to the following criterion: the value of the goal function and each solution is assigned an adequacy measure. According to the comparison of all adequate measures of the units, a decision is made which one of them will be allowed to form the next generation and with which probability in the step of selection. The selection strategy is the "virtual roulette wheel". All population units are potentially capable for reproduction. However, whether the unit will be selected depends on the value of the goal function (a sorted ranking list is formed). Finally, the virtual roulette wheel is formed according to the assigned number in the list. Conditionally, most of the "positions" in the wheel are assigned to the best units, and the worst units have no "positions". The selected units from the "virtual roulette wheel" are copied into a new generation ready for generic operation cross-over and mutation. The elitism function is embedded, enabling automatic coping (cloning) of the best unit (according to the goal function value). Thus, the danger of losing the best unit during the cloning process is avoided.

In the next step reproduction between pairs is performed, through simple copying of the units in the next generation. This is a process until the moment when cross-over and mutation happen according to the previously defined probability. The selection of units exposed to the process of cross-over is random. They are grouped and the spot of division of the genes from the two parents is random again. Through the cross-over an exchange and recombination of the genetic material is achieved which forms a new chromosome with genes from the two parents. The mutation is a change of a unit in the chromosome. The mutation is a very important factor in the evolution, because it introduces new genes in the process. This additionally increases the diversity of the units in the population. Pairs of chromosomes (parents) are exposed to the process of cross-over and the mutated units are returned in the population of the new generation. The cycle of estimation, selection and reproduction continues until a predefined acceptable behaviour level is achieved. If the termination condition is the predefined generation number, then the best unit in the last generation is the optimum. The population units (chromosomes) in each next generation are more similar to each other with convergence towards specific part of the search space.

\section{CASE STUDY NO. 1 - GA OPTIMAL DESIGN OF AN INSTRUMENT TRANSFORMER}

Instrument transformers are of great metrological significance for electrical power measurements, especially in the field of legal metrology and trade of electrical energy. The accurate metrological analysis of a $20 \mathrm{kV}$ combined instrument transformer (CIT) (voltage transformation ratio $20000 / \sqrt{3} \mathrm{~V}: 100 / \sqrt{3} \mathrm{~V}$ and current transformation ratio 100 A: $5 \mathrm{~A})$ is possible only by numerical calculation of the magnetic field distribution in the 3D domain [11]. The following four metrological parameters are relevant to the CIT:

- voltage measurement core (VMC) voltage error pu,

- VMC phase displacement error $\delta u$,
- current measurement core (CMC) current error pi,

- $\quad$ CMC phase displacement error $\delta_{i}$.

The CIT must comply with the standard IEC 61869-4 [14]. It comprises two transformer systems in complex electromagnetic coupling:

- a current measurement system with a regime close to a short circuit in the secondary winding and

- a voltage measurement system with a regime of almost unloaded transformer, i.e. open-circuit regime.

Both measurement systems are in a common housing with strong electromagnetic influence which significantly contributes to the metrological properties of the whole device. The geometry of the CIT is displayed in Figure 2.

The goal during the optimal design process of the CIT is the minimisation of its metrological parameters $p_{u}, \delta_{u}, p_{i}$ and $\delta_{i}$, as well as the VMC voltage error $p_{u 0,25}$ at 0.25 of VMC rated load, according to the specifications of the IEC standard [10] as discussed and with initial results given in [11], [12]. Also, the difference of the absolute values of the VMC voltage error at rated load and at 0.25 of the rated load should be as minimal as possible. The minimization requirements of all the metrological parameters could be contradictory. So, the process of metrological optimal design has to be performed by posing a single objective function comprising them all. This is an example of a multi objective optimisation problem. The optimization design of the $20 \mathrm{kV}$ CIT, the case study in this paper, is made through variation of eleven input constructional and geometrical variables of the CIT. The program GA maximizes the objective function, therefore, the reciprocities of the errors are considered.

The objective function can be defined with a conservative approach from a metrological point of view - the certain measurement errors expressed through the absolute values are the metrological objective function:

$$
f_{o p t 1}=\frac{1}{1+\left|p_{u}+p_{u 0,25}\right|}+\frac{1}{1+\left|p_{u}\right|}+\frac{1}{1+\left|p_{u 0,25}\right|}+\frac{1}{1+\left|p_{i}\right|}
$$

as combination of the VMC voltage error $p_{u}$ at rated load, VMC voltage error $p_{40,25}$ at 0.25 of the rated load and CMC current error $p_{i}$ at rated load. The phase displacement errors are constraints during the optimization process in order to satisfy the achieved

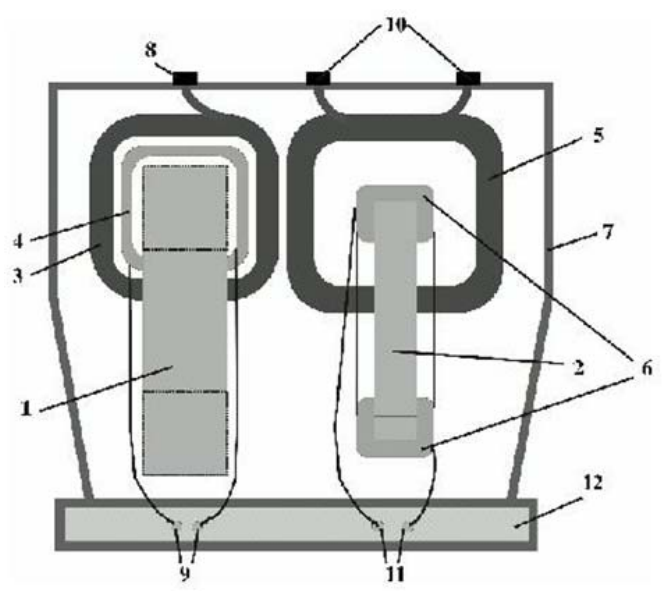

Figure. 2. Geometry of the instrument transformer
1. VMC iron core
2. $\mathrm{CMC}$ iron core
3., 4. VMC windings
5., 6. CMC windings. 
accuracy class of both measurement cores. The CIT is a highly non-linear electromagnetic system, with mutual electromagnetic influence of both cores which determines the metrological performance of the device. The GA optimal design is coupled to the results of the FEM-3D analysis of the magnetic field distribution in the CIT as in [11], [12]. Some of the initial results of the optimal design process with the goal function as defined in (1) are given in [11], [12].

According to the Guide for Expressing the Uncertainty in Measurements (GUM) [15], a statistical approach can be applied:

$$
f_{o p t 2}=\frac{1}{1+\sqrt{\left(p_{u}+p_{u 0,25}\right)^{2}+p_{u}^{2}+p_{u 0,25}{ }^{2}+{p_{i}}^{2}}}
$$

In the objective functions (1) and (2) the phase displacement error $\delta_{u}$ and $\delta_{i}$ are constraints (a control criterion of the maximal allowed values of the phase displacement errors for certain CIT accuracy classes in the GA algorithm CIT mathematical model is embedded, as in [14]). The CIT optimal design starts with the parameters derived by classical analytical design of the CIT based on the T-equivalent circuit as a CIT model given in [11], [12] by coupling with FEM-3D modelling and calculation of the main electromagnetic characteristics. The chromosome comprises 11 genes (input variables-geometrical, constructional and electrical parameters). The mapping range of the eleven GA input variables is defined according to the previously derived results by FEM-3D and the analytical transformer design, some most characteristic, are given in Table 1.

The optimal solution is derived by the following genetic parameters: cross-over probability 0.65 , mutation probability 0.03 , population size 16, maximal number of generations 30000 .

In Figures 3 to 5, the changes throughout the 30000 generations in the GA optimisation process of the current densities in the CIT windings, as input variables (genes) are comparatively displayed for both objective functions: conservative approach $f_{\text {opt } 1}$ and statistical approach $f_{\text {opt } 2 \text {. }}$

The main input variables in the calculation of the CIT metrological parameters are the equivalent T-circuit parameters, i.e. the winding's resistances and the leakage reactances.

In Figures 6 to 12, changes throughout the 30000 generations in the GA optimisation process of the CIT equivalent T-circuit

Table 1. Input variables for CIT optimal design (mapping range).

\begin{tabular}{|c|c|c|c|}
\hline Input variable & Minimum & Maximum & $\begin{array}{l}\text { Initial value } \\
\text { (analytical) }\end{array}$ \\
\hline $\begin{array}{l}\text { VMC primary winding } \\
\text { number of turns }\end{array}$ & 23584 & 24000 & 24000 \\
\hline $\begin{array}{l}\text { VMC primary winding } \\
\text { current density }\left[\mathrm{A} / \mathrm{mm}^{2}\right]\end{array}$ & 1.5 & 3.0 & 2.04 \\
\hline $\begin{array}{l}\text { VMC secondary winding } \\
\text { current density }\left[\mathrm{A} / \mathrm{mm}^{2}\right]\end{array}$ & 2.0 & 3.0 & 2.61 \\
\hline $\begin{array}{l}\text { VMC magnetic core outside } \\
\text { length }[\mathrm{mm}]\end{array}$ & 183 & 193 & 185 \\
\hline $\begin{array}{l}\text { CMC secondary winding } \\
\text { number of turns }\end{array}$ & 115 & 125 & 120 \\
\hline $\begin{array}{l}\text { CMC primary winding } \\
\text { current density }\left[\mathrm{A} / \mathrm{mm}^{2}\right]\end{array}$ & 1.0 & 1.6 & 1.36 \\
\hline $\begin{array}{l}\text { CMC secondary winding } \\
\text { current density }\left[\mathrm{A} / \mathrm{mm}^{2}\right]\end{array}$ & 2.0 & 3.0 & 2.55 \\
\hline $\begin{array}{l}\text { CMC magnetic core outside } \\
\text { length }[\mathrm{mm}]\end{array}$ & 136 & 162 & 142 \\
\hline
\end{tabular}

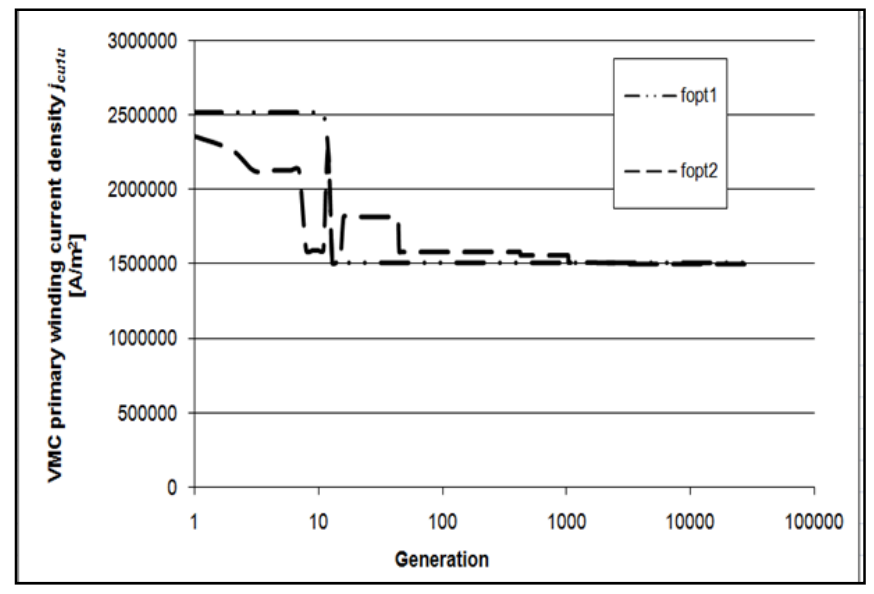

Figure. 3. Genetic algorithm changes through the generations of the VMC primary winding current density.

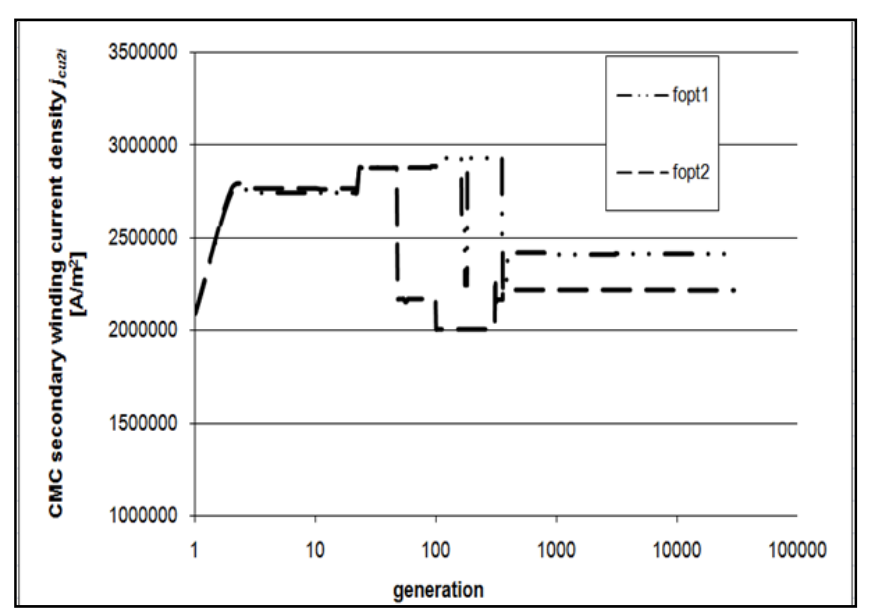

Figure. 4. Genetic algorithm changes through the generations of the CMC secondary winding current density.

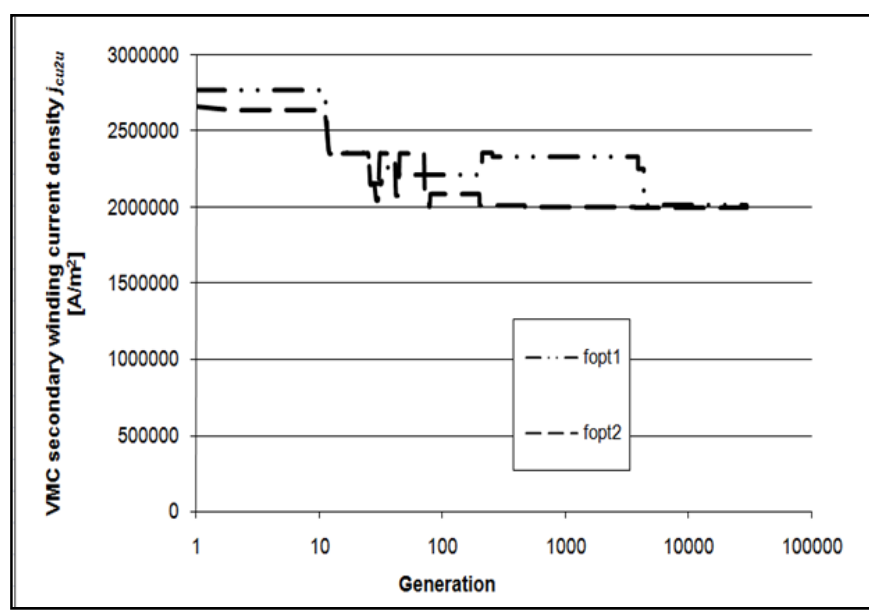

Figure. 5. Genetic algorithm changes through the generations of the VMC secondary winding current density.

parameters are comparatively displayed for both objective functions: conservative approach $f_{\text {opt } 1}$ and statistical approach $f_{\text {opt } 2 \text {. }}$

In Table 2 some of the optimal results (metrological parameters, geometrical, constructional and electromagnetic) derived by both the goal functions (1)-conservative approach and (2)-statistical approach, are compared. 


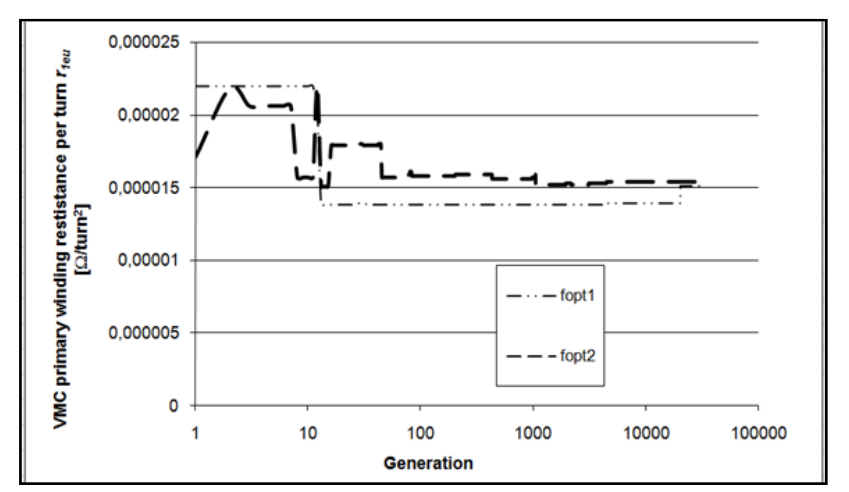

Figure. 6. Genetic algorithm changes through the generations of the VMC primary winding resistance per turn.

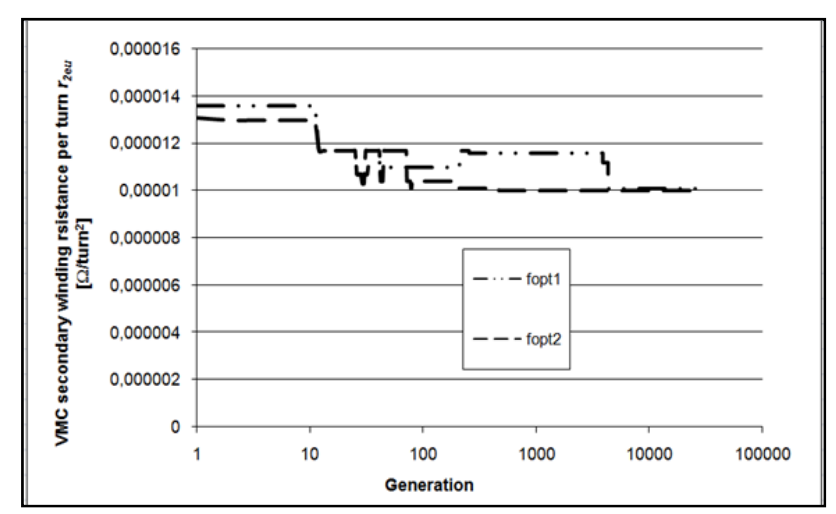

Figure. 7. Genetic algorithm changes through the generations of the VMC secondary winding resistance per turn.

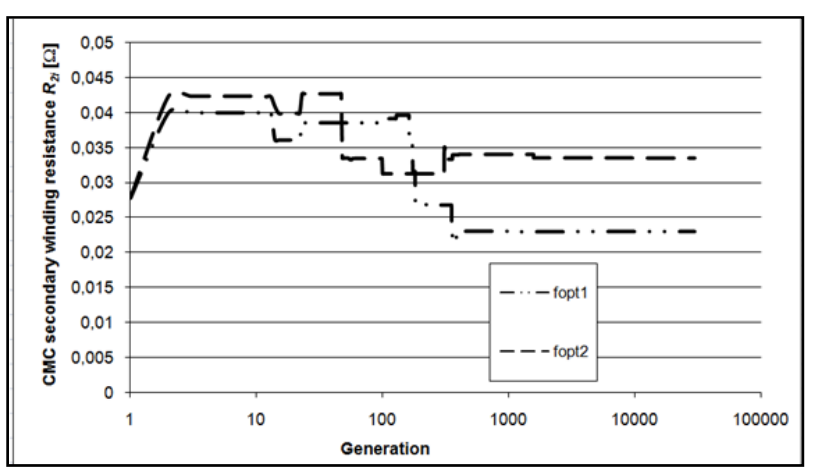

Figure. 8. Genetic algorithm changes through the generations of the CMC secondary winding resistance per turn.

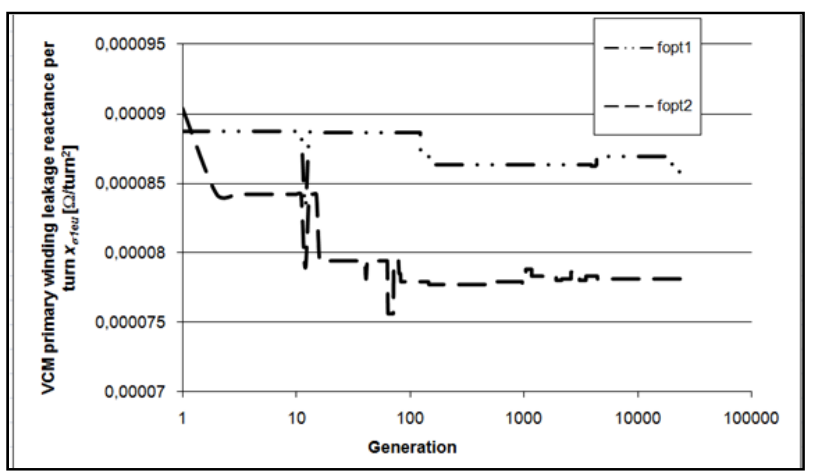

Figure. 9. Genetic algorithm changes through the generations of the VMC primary winding leakage reactance per turn.

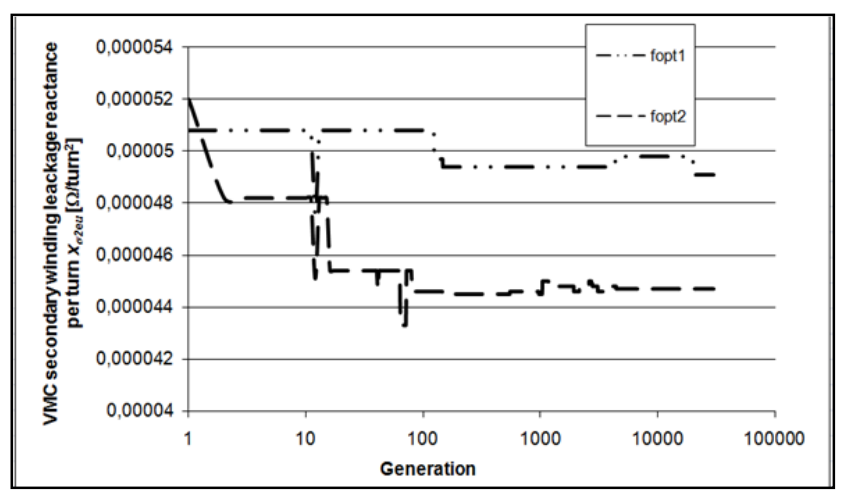

Figure. 10. Genetic algorithm changes through the generations of the VMC secondary winding leakage reactance per turn.

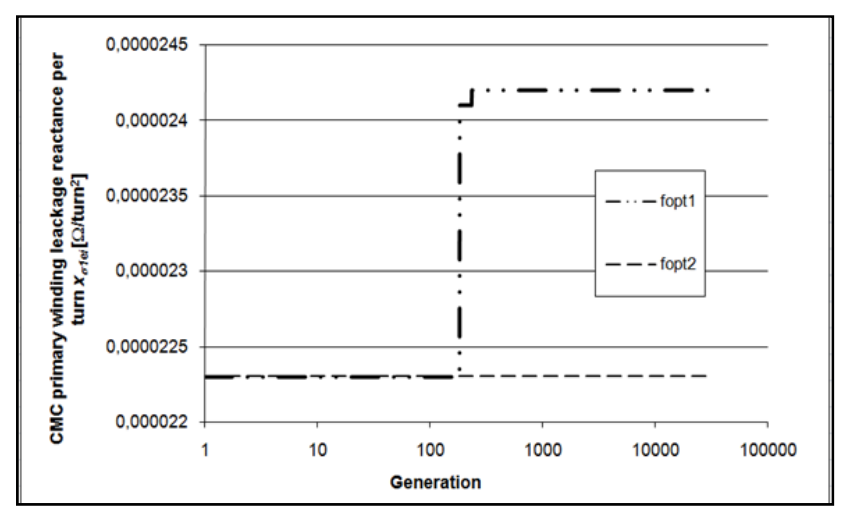

Figure. 11. Genetic algorithm changes through the generations of the CMC primary winding leakage reactance per turn.

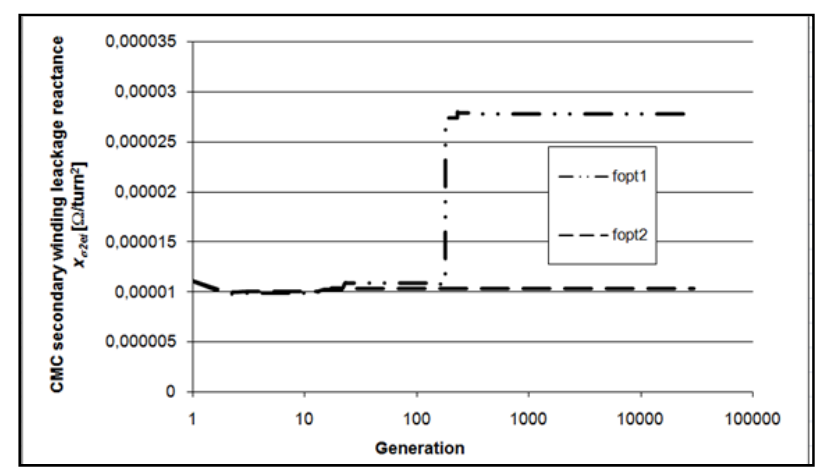

Figure. 12. Genetic algorithm changes through the generations of the CMC secondary winding leakage reactance per turn.

Table 2. Comparison of the CIT metrological, electromagnetic and constructive parameters derived by goal function with conservative and statistical approach.

\begin{tabular}{ccc}
\hline $\begin{array}{l}\text { GA derived CIT } \\
\text { parameter }\end{array}$ & $\begin{array}{c}\text { Conservative } \\
\text { approach }\end{array}$ & $\begin{array}{c}\text { Statistical } \\
\text { approach }\end{array}$ \\
\hline$p_{u}[\%]$ & -0.795 & -0.625 \\
$p_{u 0,25}[\%]$ & 0.795 & 0.855 \\
$p_{i}[\%]$ & 0.0 & $10^{-7}$ \\
$\delta_{u}[\mathrm{~min}]$ & -69.43 & -61.62 \\
$\delta_{i}[\mathrm{~min}]$ & -10.45 & 2.30 \\
$B_{m u}[\mathrm{~T}]$ & 0.424 & 0.336 \\
$B_{m i}[\mathrm{~T}]$ & 0.539 & 0.165 \\
$m_{\mathrm{Fe}}[\mathrm{kg}]$ & 35.18 & 46.58 \\
$m_{\mathrm{Cu}}[\mathrm{kg}]$ & 12.02 & 13.57 \\
\hline
\end{tabular}




\section{CASE STUDY NO. 2 - GA FOR PREDICTION OF RESISTANCE STANDARDS TIME DRIFT}

The estimation of the optimal moment for calibration of the standards is a challenge for most of the metrological laboratories [16]. The decisions are made based on the empirical experience of the laboratory staff, the behaviour history and the conditions of the standard [16]. The trend lines are usually extrapolated to predict the time-behaviour of the standards by using the classical least squares method, as in [5]. However, these models do not take into account the random nature of the calibration results and uncertainties.

In this contribution a new model of a time-drift trend line of resistance standard derived by using the Lagrange polynomial (LP), coupled with genetic algorithm optimization, is presented. The time points of the performed calibrations and the gained values of standard resistance are input data. The final model of the trend line using five input experimental values can be reduced to a polynomial of fourth order:

$R(t)=a_{0} \cdot t^{4}+a_{1} \cdot t^{3}+a_{2} \cdot t^{2}+a_{3} \cdot t+a_{4}$

where $t$ is the time, $R$ is the resistance and $a_{i}, \mathrm{i}=0, \ldots, 4$ are the LP coefficients, depending on the values of the resistance at five calibration moments. The LP model is derived on the basis of the experimental data of calibrations of $1 \Omega$ standard in time intervals from 1996 to 2005 year as in Table 3.

The goal function of the optimization process by the genetic algorithm (GA) is the minimum difference between the theoretical resistance value, derived by the LP, and the experimental value of the resistance at certain time moment (2007-04-20). The original GA program maximizes the goal function and the following goal function $f_{\text {opt }}$ is given in the optimization process:

$$
f_{\text {opt }}=\frac{1}{1+\left|R_{6}-R_{6 \exp }\right|}
$$

where $R_{6}=R_{2007}$ is the resistance of the standard derived by the LP, determined by GA, at the time moment 20. 04. 2007 and $R_{\text {6exp }}$ is the resistance of the standard derived by experimental calibration on 2007-04-20.

A verification of the gained model with known experimental data is done (for the time point 2010-06-14). The verification criteria $k$ is:

$$
k=\frac{\left|R_{7}-R_{7 \exp }\right|}{\left|s_{R 7}\right|+\left|s_{R 7 \exp }\right|}
$$

where $R_{7}=R_{2010}$ is the resistance of the standard derived by the LP, determined by GA, at the time moment 2010-06-14 and $R_{7 \exp }$ is the resistance of the standard derived by experimental

Table 3. The experimental GA input data for the LP model.

\begin{tabular}{ccc}
\hline Date & $\boldsymbol{R}[\Omega]$ & Uncert. of $\boldsymbol{R}[\Omega]$ \\
\hline $1996-09-26$ & 0.999992300 & $\pm 5 \cdot 10^{-7}$ \\
$1998-10-23$ & 0.999992450 & $\pm 3 \cdot 10^{-8}$ \\
$2000-12-28$ & 0.999992330 & $\pm 6 \cdot 10^{-9}$ \\
$2003-04-08$ & 0.999992306 & $\pm 6 \cdot 10^{-9}$ \\
$2005-05-09$ & 0.999992353 & $\pm 1 \cdot 10^{-8}$ \\
$2007-04-20$ & 0.999992349 & $\pm 1 \cdot 10^{-8}$ \\
$2010-06-14$ & 0.999992461 & $\pm 1 \cdot 10^{-8}$ \\
\hline
\end{tabular}

calibration on 2007-06-14, $s_{R 7}$ and $s_{R 7 \exp }$ are the respective uncertainties. The optimal solution is for the following genetic parameters: cross-over probability 0.65 , mutation probability 0.03 , population size 10, maximal number of generations 10000 .

The resistance standard values in the five time points derived from the process of genetic algorithm optimization are given in Table 4.

The derived GA results after 10000 generations for the Lagrange polynomial coefficients are given in Table 5.

The value of the $1 \Omega$ standard resistance in 2007 derived by the LP coupled with the GA optimization process is $R_{2007}$ $=0.999992349 \Omega$.

The verification of the method is done through comparison of the theoretical standard resistance in 2010, with the experimentally derived value through calibration. The theoretical value of the standard resistance on 2010-06-14, gained by the LP trend line and through the GA process, is 0.9999916844 , with difference of $-7.77 \cdot 10^{-7} \Omega$, which is the same order of uncertainty as the input variable with highest uncertainty $\mathrm{R}_{1996}$.

The experimental input values of the $1 \Omega$ resistance standard are compared in Figure 13 to the resistance values in different time points derived by the Lagrange polynomial coupled to the genetic algorithm optimization process.

The uncertainties of the theoretical values are compared to the measurement uncertainties of the experimental data and overlapping of the both uncertainties interval exists. The overlapping is satisfactory for the point in 2010, which is a verification point of the methodology.

Table 4. Resistance standard values at five time points derived from the GA optimization process.

\begin{tabular}{ccccc}
\hline $\boldsymbol{R}_{\mathbf{1 9 9 6}}[\Omega]$ & $\boldsymbol{R}_{\mathbf{1 9 9 8}}[\Omega]$ & $\boldsymbol{R}_{\mathbf{2 0 0 0}}[\Omega]$ & $\boldsymbol{R}_{\mathbf{2 0 0 3}}[\Omega]$ & $\boldsymbol{R}_{\mathbf{2 0 0 5}}[\Omega]$ \\
\hline 0.99999245 & 0.99999242 & 0.9999923 & 0.9999923 & 0.99999235 \\
\hline
\end{tabular}

Table 5. The GA output data for the LP model.

\begin{tabular}{lc}
\hline LP coefficient & GA derived value \\
\hline$a_{0}\left[\Omega /\right.$ month $\left.^{4}\right]$ & 0.00000000000 \\
$a_{1}\left[\Omega /\right.$ month $\left.^{3}\right]$ & 0.00000000000 \\
$a_{2}\left[\Omega /\right.$ month $\left.^{2}\right]$ & -0.00000000025 \\
$a_{3}[\Omega /$ month $]$ & 0.00000000378 \\
$a_{4}[\Omega]$ & 0.999992438 \\
\hline
\end{tabular}

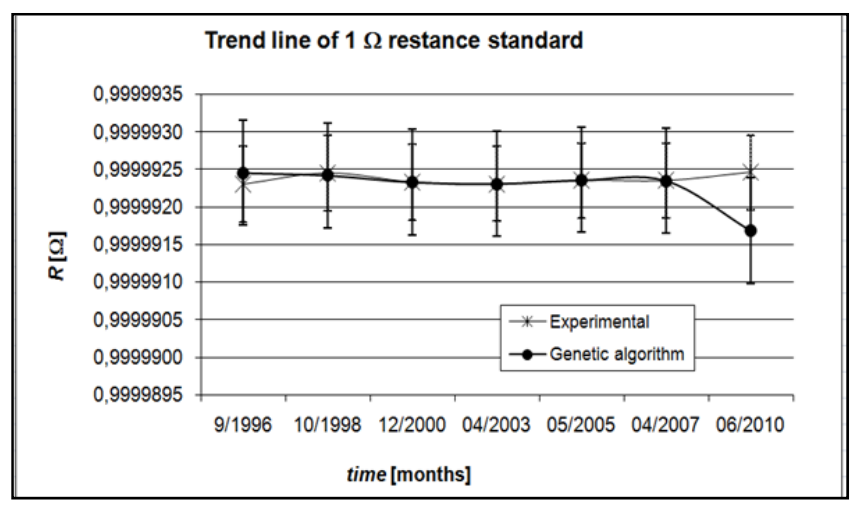

Figure. 13. Comparison of the GA derived LP time-trend line and the experimentally derived time-drift of $1 \Omega$ resistance standard. 


\section{CONCLUSIONS}

In this paper the stochastic optimization method-genetic algorithm is applied for gaining a solution of two different, but challenging problems in metrology of electrical quantities:

1. Reduction of errors and uncertainties during the design process of an instrument transformer;

2. Prediction of the time-drift of a resistance standard.

One original and universal GA software solution is used for two problems of diverse nature. The derived results are satisfactory and verified. It can be concluded that lots of options exist, i.e. the genetic algorithm further to be applied for gaining other optimal solutions in different metrology areas and problems of stochastic nature.

\section{REFERENCES}

[1] M. Cundeva-Blajer, "Optimization Methods in Metrology of Electrical Quantities", Proc. of 21 $1^{\text {st }}$ IMEKO World Congress, Aug. 30-Sept. 4, 2015, Prague, Czech Republic, pp. 1903-1908.

[2] T. N. Siraya, "Regression and Confluent Algorithms and Models For Construction Of Functions In Measurements", Proc. of $21^{\text {st }}$ IMEKO World Congress, Aug. 30-Sept. 4, 2015, Prague, Czech Republic, pp. 2047-2051.

[3] N. Makarava, S. Eichstaedt, "Stochastic modelling for state estimation in medium-voltage electric grids", Proc. of $21^{\mathrm{s}}$ IMEKO World Congress, Aug. 30-Sept. 4, 2015, Prague, Czech Republic, pp. 1997-2001.

[4] A. L. Shestakov, G. A. Sviridyuk, A. V. Keller, "Optimal Measurements", Proc. of $21^{\text {st }}$ IMEKO World Congress, Aug. 30Sept. 4, 2015, Prague, Czech Republic, pp. 2072-2281.

[5] P. Mostarac, R. Malarić, H. Hegeduš "Extrapolation of Resistance Standard Values between Calibrations", Conf. Proc. of the IX Nat. Conf. with Int. Part. ETAI, Ohrid, Macedonia, CD ROM E2-2, 2009.

[6] M. Cundeva-Blajer, L. Arsov "Prediction of Resistance Standards Time Behaviour by Stochastic Determination of Lagrange Polynomial", Series on Advances in Mathematics for Applied Sciences-Vol.84, Advanced Mathematical and Computational Tools in Metrology and Testing IX, World Scientific Publishing Co. Pte. Ltd, 2012, pp. 102-109.

[7] C. E. Matthews, L. Wright, X-S Yang "Metrology and Design with Continuous Models: tools for optimisation, sensitivity and uncertainty evaluation", Series on Advances in Mathematics for Applied Sciences-Vol.84, Advanced Mathematical and Computational Tools in Metrology and Testing IX, World Scientific Publishing, 2012, pp. 260-267.

[8] K. K. Semenov, G. N. Solopchenko "Inverse Problems in Theory and Practice of Measurements and Metrology", Series on Advances in Mathematics for Applied Sciences-Vol.86, Advanced Mathematical and Computational Tools in Metrology and Testing X, World Scientific Publishing Co. Pte. Ltd, 2015, pp. 330-339.

[9] M. Cox, P. Harris "Polynomial Calibration Functions Revisited: Numerical and Statistical Issues", Series on Advances in Mathematics for Applied Sciences-Vol.86, Advanced Mathematical and Computational Tools in Metrology and Testing X, World Scientific Publishing Co. Pte. Ltd, 2015, pp. 916.

[10] G. Cvetkovski, L. Petkovska, S. Gair, "Specific power as objective function in GA optimal design of permanent magnet disc motor", COMPEL: Int. Jour. for Computation and Mathematics in Electrical and Electronic Engineering 29, Iss. 4, 2010, pp. 964-973.

[11] M. Cundeva-Blajer, L. Arsov „Computer Aided Techniques for Estimation and Reduction of Electromagnetic Measurement Devices Uncertainties“, Int. Journal of Metrology and Quality Engineering, Vol. 2, EDP Sciences, Paris, 2010, pp. 89-97.

[12] M. Cundeva-Blajer, L. Arsov "Influence of the Objective Function in the Process of Optimal Design of an Electromagnetic Measurement System”, Int. Journal of Applied Electromagnetics and Mechanics Vol. 39, IOS Press, Amsterdam, 2012, pp. 705-710.

[13] D. Filipovic-Grcic, M. Pljak, Z. Stih, "Optimization of the PaperOil Insulation of High Voltage Inverse Current Transformers Using Genetic Algorithm", Proc. of 13th Int. Symp. on Electromagnetic Fields in El. Eng. ISEF'07, Prague, (paper ID 065 on a CD ROM), 2007.

[14] IEC 61869-4, Edition 1.0, 2013-11: Instrument transformers, Part 4: Additional requirements for combined transformers, Geneva, 2013

[15] Expression of the Uncertainty of Measurements in Calibration, (GUM), JCGM 100 with member organizations (BIPM, IEC, ILAC, OIML, ....), 1999.

[16] ILAC-G24:2007 / OIML D 10:2007 (E) Guidelines for the determination of calibration intervals of measuring instruments, 2007. 